\title{
A Light-weight, Temporary File System for Large-scale Web Servers
}

\author{
Jun Wang and Dong Li \\ Computer Science and Engineering Department \\ University of Nebraska Lincoln \\ Lincoln, Nebraska \\ email:\{wang,li\}@cse.unl.edu
}

September 24, 2003

\begin{abstract}
Several recent studies have pointed out that file I/Os can be a major performance bottleneck for some large Web servers. Large I/O buffer caches often do not work effectively for large servers. This paper presents a novel, light-weight, Temporary File System called TFS that can effectively improve $\mathrm{I} / \mathrm{O}$ performance for large servers. TFS is a more cost-effective scheme compared to the full caching policy for large servers. It is a user-level application that manages files on a raw disk or raw disk partition and works in conjunction with a file system as an I/O accelerator. Since the entire system works in the user space, it is easy and inexpensive to implement and maintain. It also has good portability. TFS uses a novel disk storage subsystem called Clusterstructured Storage System (CSS) to manage files. CSS uses only large disk reads and writes and does no have garbage collection problems. Comprehensive trace-driven simulation experiments show that, TFS achieves up to $160 \%$ better system throughput and reduces up to 77\% I/O latency per URL operation than that in a traditional Unix Fast File System in large Web servers.
\end{abstract}

\section{Introduction}

Recent studies [1, 2, 3] have shown that disk I/O is one of the important performance bottlenecks for large Web servers. Full caching (cache all Web files in very large RAM) is one possible solution but it would be very expensive at high throughput levels. This is because that the file system size often increases linearly with the server throughput [1].

The exponential growth of the WWW and its architecture evolution result in the changes of salient characteristic features of Web visitors [4]. These changes make the Least Recently Used (LRU) cache management policy less effective. Other recent techniques such as content distributed network $(\mathrm{CDN})$, reverse proxy server and Greedy-Dual algorithm [12] are not much helpful in eliminating I/O problems.

To address this problem, we argue that, rather than caching a huge file data set in RAM, we should pay more at- tention to the efficiencies of secondary storage management. We aim at designing a cost-effective, high-performance I/O subsystem with only a modest I/O buffer cache size (such as $256 \mathrm{MB}$ ) to replace a full caching scheme (which requires 4 GB of RAM or more) for large Web servers.

Currently most Web servers are designed to run on top of a general purpose file system. Such a design has several inherent performance disadvantages:

1. Small documents dominate the Web server access patterns $[5,6]$ but FFS and other conventional file systems cannot efficiently handle small files $[7,8,9,10]$.

2. Read requests dominate the file system traffic in Web server workloads [11]. Unfortunately traditional LRU algorithm for I/O buffer cache does not work well under Web server workloads because web proxies and client browsers caches have filtered out major temporal locality from Web server accesses [4]. Although there are many results published on improving file system write performance $[7,9,8,12]$, relatively few has been done to improve read performance.

3. Web server workloads often demonstrate good spatial locality $[13,2,3]$, which implies that prefetching is a attractive scheme in file system design. However, Native File Systems (NaFS) has a very limited prefetching ability that prefetch blocks one by one within a file.

4. The meta-data overhead also poses a serious problem for native file systems.

Therefore, we propose to let Web servers manage their own data and meta-data on raw disks and by-pass NaFS. For reasons of compatibility, simplicity and easy implementation, we propose a new Temporary File System (TFS) to solve above problems. TFS works in front of a NaFS to service repeated file accesses while NaFS still handles advanced issues like file creations, invalidations, security, protection and etc. When a file is read for the first time, TFS intercepts a file copy from the NaFS (resides on a raw disk partition). All later accesses to this file will be handed over to TFS until 
the file is modified or deleted. The entire process is completely transparent to users.

\section{The design of Temporary File Sys- tem}

\subsection{TFS System Architecture}

The system consists of the following major components: a cluster-structured storage system (CSS), an in-memory file lookup table, a disk cluster table, a TFS application buffer cache and a locality-based grouping algorithm.

Figure 1 shows the illustration of the system architecture.



Figure 1: TFS System Architecture

When the Web server receives a URL request, it will check in-memory data structures and decide how to handle this request. The Web server system will work in the following steps: First, the system checks the I/O buffer cache. If the requested file is in the I/O buffer cache and is still valid, the file is sent to the client directly. Second, in case of an I/O buffer cache miss, the system passes the URL to the TFS. The TFS calculates its ID and searches the in-memory file lookup table. Upon a hit in the TFS buffer cache, the TFS serves the request right away. Third, if the file misses in the TFS buffer cache but is saved in the CSS, TFS will load the entire cluster or additional related clusters (this is a large file, see more details in section 2.3.1) containing the requested file into the TFS buffer cache, as shown in Figure 1. Since the clusters may contain many other files, these files are also prefetched into TFS buffer cache. Because the locality grouping algorithm puts files into clusters according to their access locality, the prefetched files are most likely to be accessed soon. If the TFS buffer cache is full, the grouping process will start to group LRU files into one or multiple clusters and evict them to the disk. Lastly, if the requested file is not in both buffer caches and the CSS, the system knows that the file is accessed at the first time. The system will read this file from the NaFS disk partition and copy it to the TFS buffer cache.

$\mathrm{NaFS}$ is only used for initial accesses of files. Its I/O buffer cache is very small and acts more like a buffer rather than a "cache". All repeated file accesses are handled by the TFS. In the following subsections, we describe several important components of TFS.

\subsection{In-Memory File Lookup Table}

TFS tries to keep all necessary meta-data in RAM such that searching and updating meta-data do not generate any disk I/O in TFS. When a URL request comes in, the TFS needs to quickly decide whether this URL document is cached or not, and if yes, where the document is located. We use a hash table to realize such functions. Each cached file in TFS has one entry, which contains the MD5 checksum of the URL path as the file ID, the disk cluster address of the file (cluster ID), or the location pointer to the buffer cache if the file is in RAM. TFS uses a bit to indicate whether the requested URL file is in RAM ("1") or on disk ("0"). For performance reasons, one file may be duplicated in multiple clusters. We use a "cluster array"(see more in section 2.3.1) to record all cluster IDs that contain this file. Figure 2 shows the details of the lookup table.

\begin{tabular}{|c|c|c|}
\hline MD5 Checksum (16Bytes) & $\begin{array}{c}\text { Cluster Index Array(4B*N) } \\
\text { or Pointers to a RAM Adr. }\end{array}$ & MEM(1Bit) \\
\hline 00001111222233334444555566667777 & 1200 & 0 \\
\hline 12345678 90ab cdef 1234 5678 90ab cdee & 0000 AA00 & 1 \\
\hline 12345678 90ab cdef 1234 5678 90ab cdef & $1600,1666,1601$ & 0 \\
\hline & & \\
\hline 195c 77d1 ceff ed8e 2d2f e0fb 7634 6180 & 32688 & 0 \\
\hline
\end{tabular}

Figure 2: The In-memory File Lookup Table

\subsection{Cluster-structured Storage System}

CSS is the key to the whole design. CSS divides its disk space into large, fix-sized clusters, which are the basic units for disk reads and writes. The cluster size is normally $64 \mathrm{~KB}$ or $128 \mathrm{~KB}^{1}$. Such large-only I/O maximizes the use of disk bandwidth and improves the CPU utilization.

2.3.1 Disk layout. Figure 3 shows the disk layout of CSS. Each disk cluster may contain multiple small files (which is the common case). On the other hand, a large file can span multiple clusters.

\footnotetext{
${ }^{1}$ From the experiments, we found a $64 \mathrm{~KB}$ or $128 \mathrm{~KB}$ cluster size achieves the best performance.
} 
The file meta-data consists of the following fields that are stored together with the file data: 1) The URL path of the cached file, obtained from the HTTP request and records the full path name of this file in Web servers. 2) The File Size. 3) The Last-Access-Time of the file. 4) A Cluster Array. If a file is larger than a cluster, it will span over multiple clusters. This array records the cluster IDs of the remaining clusters of this file if the file size is larger than one cluster. The array has 4 entries. If the cluster size is $64 \mathrm{~KB}$, then the maximum file size allowed is $4 \times 64 \mathrm{~KB}=256 \mathrm{~KB}$. The array contents are ignored if the file is smaller than one cluster.

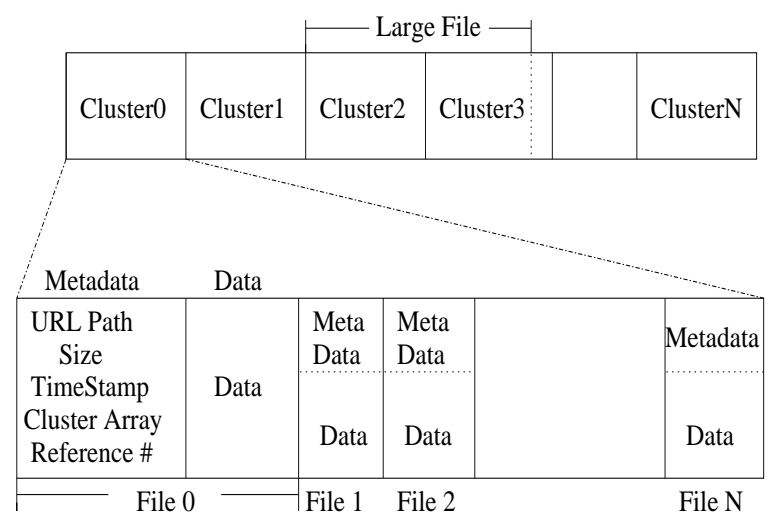

Figure 3: Disk-layout Table of TFS

In addition to the normal data clusters, CSS pre-allocates a super-block at the beginning of the disk to save important information such as the cluster size, the pointers to the disk copies of file lookup table and disk cluster table.

2.3.2 Writes. The write operation in CSS is simple. TFS only writes files to the CSS disk space when the buffer cache is full. Files are first written into the group buffer and the write requests are considered done. When the group buffer fills up, the grouping process would group affiliated files together into one or more clusters and write them to the raw disk, in a single large disk write. As in the case of Log-structured File System [14, 7], such large writes in CSS are very efficient.

2.3.3 Reads. The read operation in CSS is very different from those in other file systems such as LFS or FFS. Because clusters are the basic I/O unit in CSS, when reading a file, the entire cluster that contains that file is read, in a single large disk $\mathrm{I} / \mathrm{O}$, into the memory.

There are two important reasons of choosing such a design:

\section{- Affiliated Large Reads}

First, reading an entire cluster that contains many files, instead of a single file, is in fact doing prefetching with very few extra overhead because the disk seek and rotational latency, which are independent of request sizes, dominate the disk access time.
Since our system groups files into a cluster according to their locality, it is very likely that when one file in the cluster is accessed, some other files in the cluster will be accessed soon.

\section{- No Meta-data I/O Overhead}

Second,the file meta-data is stored together with file data as an atomic storage unit on the disk. It will also be read into the memory at the same time when the file data is read into the buffer cache. Any future accesses, updates or invalidations to the file meta-data will be done only in memory.

2.3.4 Invalidation. When NaFS invalidates a file (i.e, the file is stale), it will ask TFS to check its in-memory file lookup table whether this stale file is in TFS. If the stale file is in the TFS buffer cache, TFS simply invalidates it in memory. If it is in CSS, TFS will invalidate all clusters that contain the stale file. The overhead is not a concern since we see very few updates of documents (normally less than 1\%) in all of the three Web server workloads in this paper. TFS clears the corresponding entries in the disk cluster table and entries in the file lookup table. Note that invalidations are performed only on the in-memory data structures rather than those on the disk.

This strategy has several advantages: first, the whole invalidation process is done in RAM and no disk I/O is involved. Second, this method does not generate any garbage holes on the disk, which is the major problem of LFS. Garbage is generated because individual files in a disk cluster (or segment in LFS) are invalidated. Since TFS uses clusteronly invalidation, garbage will never be generated.

When the invalidated files are accessed again, they will be loaded from NaFS and copied into the group buffer, and later be regrouped and written to a new cluster on CSS.

2.3.5 Large Files. Large files are not common in Web server workloads. In TFS, if a file is larger than the size of the cluster, it will span over several clusters. These clusters do not need to be contiguous on the disk. We use a "cluster array" to indicate the cluster numbers of the remaining clusters. TFS will always have good performance when reading and writing large files across several clusters. The minimal disk request size is the cluster size, and the request size will be larger if clusters are stored on the disk contiguously. But FFS and other traditional file systems may use small and expensive I/Os to handle these files if the disk is fragmented.

2.3.6 Disk Cluster Table. CSS uses an in-memory Disk Cluster Table, which is quite small (less than $800 \mathrm{k}$ even data capacity in CSS reaches $10 \mathrm{~GB}$ ), to manage clusters in the system. Each cluster has an entry that includes the following fields: 1) The Last-access-time since the last access or update. 2) A reference counter. This is the total reference number of the cluster. 3) The valid status. 
This flag indicates if the cluster is "empty" or "valid".

\subsection{TFS Buffer Cache and its Group Buffer}

The TFS buffer cache stores active files in RAM. The group buffer is a logical region of the TFS buffer cache. It is used to group files into large clusters being written to the disk later. Our experiments show that a group buffer with one-tenth size of TFS buffer cache is good enough to find associated files and perform efficient grouping.

TFS maintains a visiting client list (VCL) for every file, which records the IP addresses of all the accessing clients. If the file is in the group buffer and another client requests the same file, TFS will add the IP address of this new client to the requested file's VCL. The grouping algorithm later will use the visiting clients' information to find locality. This is because different clients may request the same file in a short period and thus the requests from different clients are interleaved. Since operations associated with VCL are done only in RAM, the overhead is quite small.

\subsection{Locality-based Grouping Algorithm}

One of the key assumptions during the design of TFS is that files reside in the same cluster have good access locality. Previous studies have shown that Web accesses indeed have good spatial locality in every client access pattern [6] and prefetching is more beneficial to the system than LRU caching [3]. Based on these results, we develop an efficient and effective algorithm that can discover the locality. The grouping process iteratively uses the algorithm listed in Table 1 until one or multiple clusters in the group buffer are produced or all HTML files are scanned.

In the experiments, the write buffer size is equal to the integer part of (leading-file-size/cluster-size)+1. A group is considered to have good affinity if it contains four or more files, or if the total grouped size is larger than $32 \mathrm{~KB}$ (half of one cluster size).

\section{Experimental Methodology}

We used HTTP trace-driven simulation to evaluate the TFS performance. We assume a Web server is running on top of a baseline file system or TFS. For the baseline system, we choose a Unix FFS, mounted on the asynchronous mode. FFS in the asynchronous mode has better performance than regular FFS, since it uses asynchronous writes for metadata updates. For our new design, we assume a TFS running together with a FFS that acts as the NaFS. Without special explanation, FFS means FFS -async in the remaining parts.

\author{
Grouping()\{ \\ if(A static HTML file cannot be found in LRU list)\{ \\ This grouping is not successful;Return; \\ \}else \{ \\ Allocate a write buffer; \\ Move this file to write buffer; \\ Record this file as the leading HTML file; \\ \} \\ Find all the files in the LRU list which have \\ the same client IP address of the leading HTML \\ file and move them to the write buffer; \\ if(The grouped buffer have a good affinity)\{ \\ Flush the data from the write buffer to the \\ disk (or disk partition) of TFS; \\ This grouping is successful;Return; \\ \}else \{ \\ Tag the leading HTML files as non-group candidate; \\ This grouping is not successful;Return; \\ \} \\ \}
}

Table 1: Pseudo code for Grouping algorithm in TFS

\subsection{Baseline System}

The baseline system uses a FFS simulator that is ported from the BSD Unix distribution. The FFS simulator is built on top of the DiskSim [15]. We chose a Quantum Atlas 10K 9.1 GB as the basic model (the largest model provided by DiskSim). It has $40 \mathrm{MB} / \mathrm{sec}$ date transfer rate and $4.7 \mathrm{~ms}$ seek time.

The FFS simulator emulates FFS cylinder groups, FFS fragments management (4KB disk block sizes and $1 \mathrm{~KB}$ fragment sizes), and a directory name look-up cache to reduce the overhead from meta-data lookup. The name cache hit rate is about $70 \%$ in the experiments. The FFS buffer cache replacement policy uses the representative Greedy-Dual algorithm [12].

\subsection{TFS System Simulator}

The TFS system simulator consists of a TFS simulator and a NaFS (FFS) simulator. To ensure fairness, the I/O buffer cache size of the baseline system (FFS) is equal to the sum of the I/O buffer cache size of NaFS and the TFS buffer cache size.

In order to simulate the file system performance precisely, we need to restore the file system with the original Web documents. We re-built the original disk layout by scanning all files on the Web traces. Those files that are not in the log traces can not be included. We parsed the URL information of directory, sub-directory and files for all HTTP requests and rebuilt the file system tree on the disk. Files with the same directory name are put logically contiguous on the disk. 
Different directories are allocated by their last-access-times. The resulting disk layout is a best-case layout without fragmentation.

\subsection{Workload Models}

We obtained a very recent Web trace, UCBSep01, from the HTTP server of the Computer Science Department of University of California at Berkeley (http://www.cs.berkeley.edu/logs/http/2001/) and two other traces, World-Cup98 and ClarkNet, from the Internet Achieve [16]. Table 2 shows the details of these traces.

These access log traces consist of a comprehensive list of information, some of which are not supported by current Web servers at all. We simulate only those requests whose replies are valid as specified in HTTP 1.1 [17]. We filter out all CGI dynamic document requests, and other unrelated TCP requests like TCP_DENIED.

In order to measure the system performance in the stable state and avoid the transient effect, we used the first 200,000 requests to warm-up the system and started measuring performance after that point.

We evaluate large Web caching architectures whose I/O buffer cache has more than 1 GB RAM capacity. The UCBSep01 trace is large enough for this purpose since its total unique data-set size is $4.8 \mathrm{~GB}$. However, the other two traces, Cup98 and ClarkNet, are too old to generate enough requests as seen in today's large-scale Web servers.

We duplicate all original requests from the original log, rename the file names but keep other HTTP attributes untouched, and insert these pseudo requests into the original log. This method is simple but does not lose much reality and correctness because the enlarged trace density reflects the fact that: current Web server host machines are more powerful, and the Web surfing population is increasing very quickly. Table 3 shows the modified features of pseudo traces.

Table 3: Characteristics of Two Large Pseudo Web Traces

\begin{tabular}{|c|c|c|}
\hline Traces & P-Cup98 & P-ClarkNet \\
\hline Total requests(K) & $17,292,062$ & 29,409 \\
\hline Total Bytes Transferred(GB) & 68,865 & 276 \\
\hline Total Distinct Bytes (MB) & 4,100 & 4,149 \\
\hline
\end{tabular}

\section{Simulation Results and Perfor- mance Analysis}

In this section we compare the I/O performance of TFS with that of the baseline system (FFS) in large Web servers. We use throughput, latency and numbers of I/Os as the performance metrics. We define the Web Server throughput as the number of processed URL requests per second (ops/sec). URL latency equals to the sum of URL I/O latency and CPU processing time (including idle time). We assume that the CPU processing time is $0.5 \mathrm{~ms}$ with a $95 \%$ confidence level.

\subsection{Simulation Results}

Figures 4 (A) shows the Web server throughput results of two systems. Figures 4 (B) shows the improvement of TFS throughput compared to that of FFS in three traces. As shown in Figures 4 (B), TFS achieves a $20-160 \%$ better system throughput than FFS does. When the I/O buffer cache size increases, the speedup decreases since more data can be cached in the FFS buffer cache. However, even when the Web server has 3 GB cache size, TFS still has achieved $20 \%$ better improvement than FFS. The reason is that TFS is a much more efficient I/O subsystem than FFS and can utilize memory and disk bandwidth more efficiently.

Figure 5(A) compares the average disk latency per URL request, calculated by dividing the total disk I/O latency by the total request number. Figure 5(B) shows the speedups of TFS over the baseline system. Again, we can clearly see that TFS makes significant performance improvements over FFS, reducing the I/O latency per URL request by $18-65 \%$.

We also calculated the total buffer cache hit rate of TFS and the I/O buffer hit rate of FFS. Figures 6 (A) shows the buffer cache hit rate in two systems. Similarly, Figures 6 (B) calculates the TFS cache hit rate improvement over that of FFS. TFS has a much higher cache hit rate than FFS. Even when the buffer cache is $3 \mathrm{~GB}$, we still see around $10 \%$ better improvement in TFS.

\subsection{Performance Analysis}

It is important to analyze why TFS achieves such a significant improvement over FFS.

In the I/O buffer cache, for each URL request, a cache miss will generate one file read. When FFS reads a Web document from the disk, it also generates multiple small disk I/Os in case of a name cache miss: several reads for multiplelevel directory inodes, one read for file inode, several writes for directory inodes and file inodes (to update the file access time), and one or more reads for the file data itself. Although the name cache of FFS can eliminate some reads to the inodes, the overhead is still excessively high. The name cache also has to compete with other meta-data and data caches for RAM.

In TFS, one disk file read will generate less than one disk read. For any TFS disk hit, by searching the in-memory file lookup table, we can locate the disk cluster that contains the requested file directly. The entire cluster, which contains multiple Web documents, is then read into the I/O buffer cache in one single disk read. Because Web accesses have strong locality, and because our algorithms put requested files with good affinity into the same cluster, it is very likely 
Table 2: Characteristics of Real-world Web Traces

\begin{tabular}{|c|c|c|c|}
\hline Properties & UCBSep01 & Cup98 & ClarkNet \\
\hline Location & U. of CA, Berkeley. CS HTTP Server & Santa Clara, Paris etc. & Baltimore-Washington \\
\hline Access log Duration & Sep.1-Oct.1,2001 & May 1-July 23,1998 & Aug. 28-Sep. 11,1995 \\
\hline Total requests & $5,413,271$ & $1,352,804,107$ & $2,940,873$ \\
\hline Total Bytes Transferred(MB) & 128,056 & $4,991,000$ & 27,592 \\
\hline Total Distinct Bytes (MB) & 4,805 & 307 & 414.9 \\
\hline Mean File Size(bytes) & 26,040 & 15,524 & 13,497 \\
\hline Median File Size(bytes) & 4,912 & 4,674 & 1,994 \\
\hline
\end{tabular}
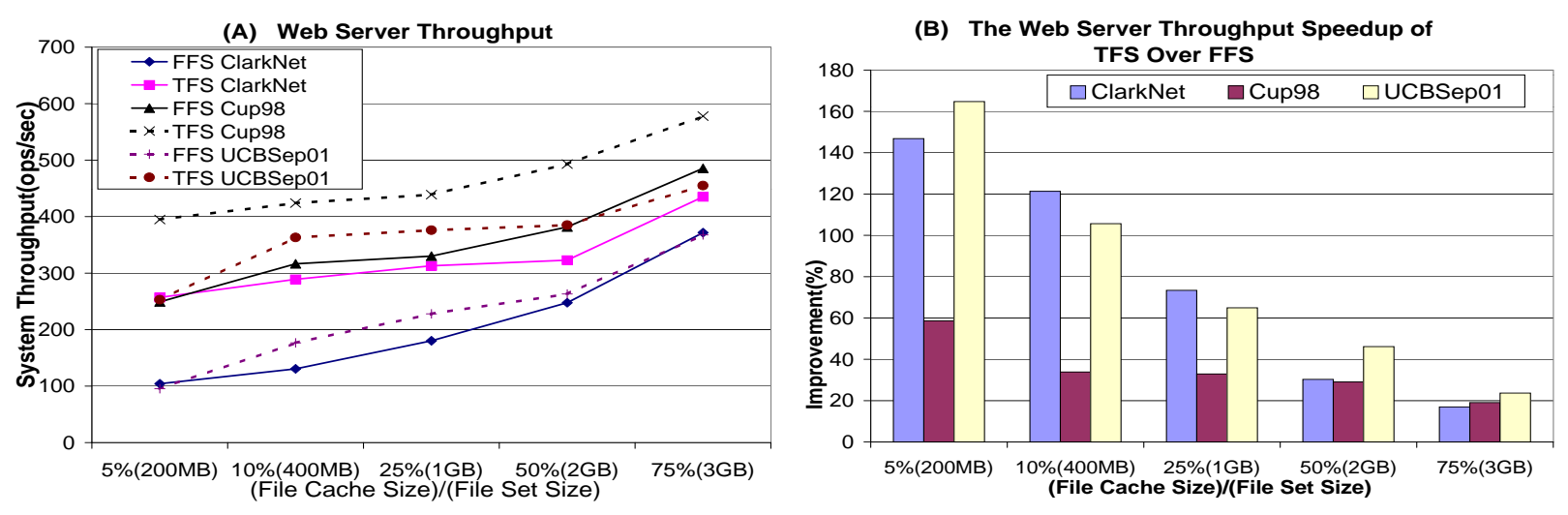

Figure 4: System Throughput between TFS and FFS

that some of the files fetched with the cluster will be accessed in the near future. Since these files are already in RAM, there are no need to read them from the disk again.

TFS achieves 10-68\% higher buffer cache hit rates than those of FFS, indicating that our locality-grouping algorithm works well.

\section{Related Work}

TFS shares some insights with its sister project called UCFS [18]. The main differences are TFS is optimized for Web servers that are almost read-only, while UCFS is for proxy servers, which see heavy write traffic. In addition, since Web servers and proxy servers see very different access patterns, data structures are very different in two systems.

Shriver and et al. described the design of Hummingbird, a light-weight file system for Web proxies [19]. There are several different points between Hummingbird and TFS: Hummingbird works for Web proxy servers while TFS services large Web servers. Their file deletion and invalidation methods are different. Hummingbird may suffer penalty of garbage collection while TFS does not have any garbage left on the disk and can always provide high I/O performance.

Some researchers also tried to improve Unix file system performance for Internet servers [20, 21]. Kaashoek et al. introduced the concept of Server Operating Systems [22]. While having good performance, such specialized OSs are difficult to design, implement and port because they are in the kernel. The Bullet server [23] is the file system for Amoeba, a distributed operating system. It stores file metadata contiguously with the file. But they do not consider clustering technique.

Several papers discussed designs of temporary file systems and memory-only file systems[24, 25]. These systems mainly try to store data in memory in order to achieve high performance, while TFS tries to keep most data on disk and still have good performance. Also, the memory-only file system and tmpfs are both implemented in the kernel space while our TFS works in the user address space.

\section{Conclusions}

This paper presents a novel light-weight, temporary file system called TFS for large Web servers. The system has the following significant advantages over traditional approaches: user-level implementation to gain good portability, CSS design together with the grouping algorithm significantly improve read performance, completely eliminating the I/O overhead caused by meta-data updates and no garbage collection problem to degrade performance. Extensive simu- 

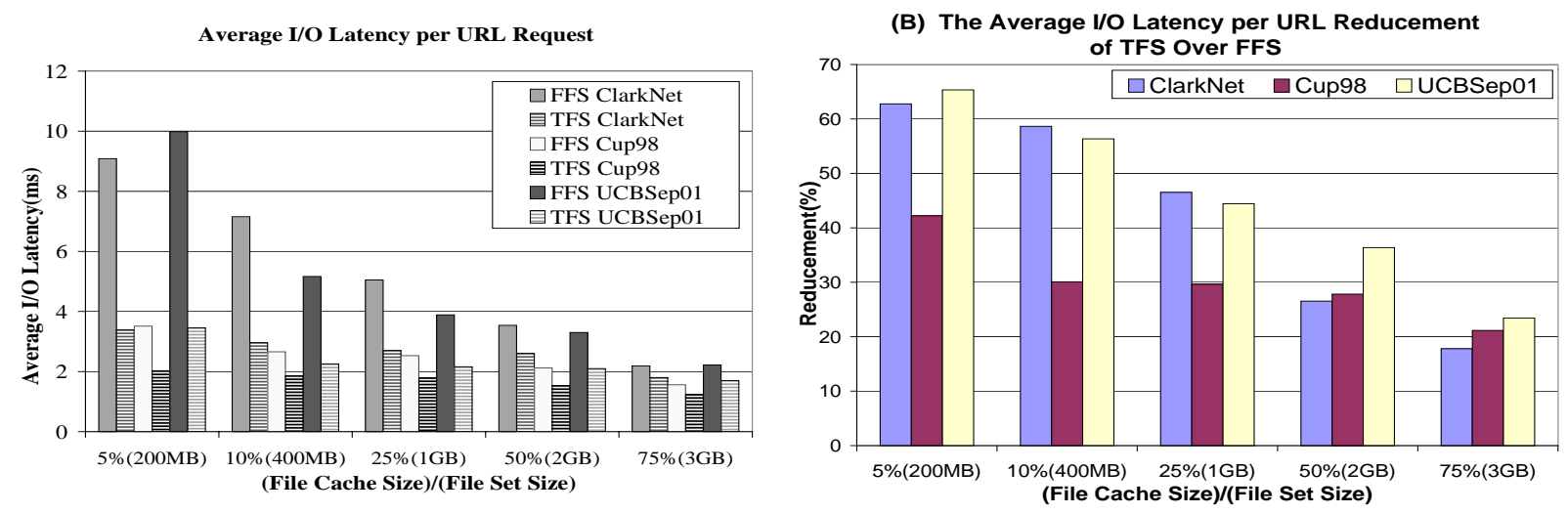

Figure 5: Average Disk I/O Latency per URL Request between TFS and FFS
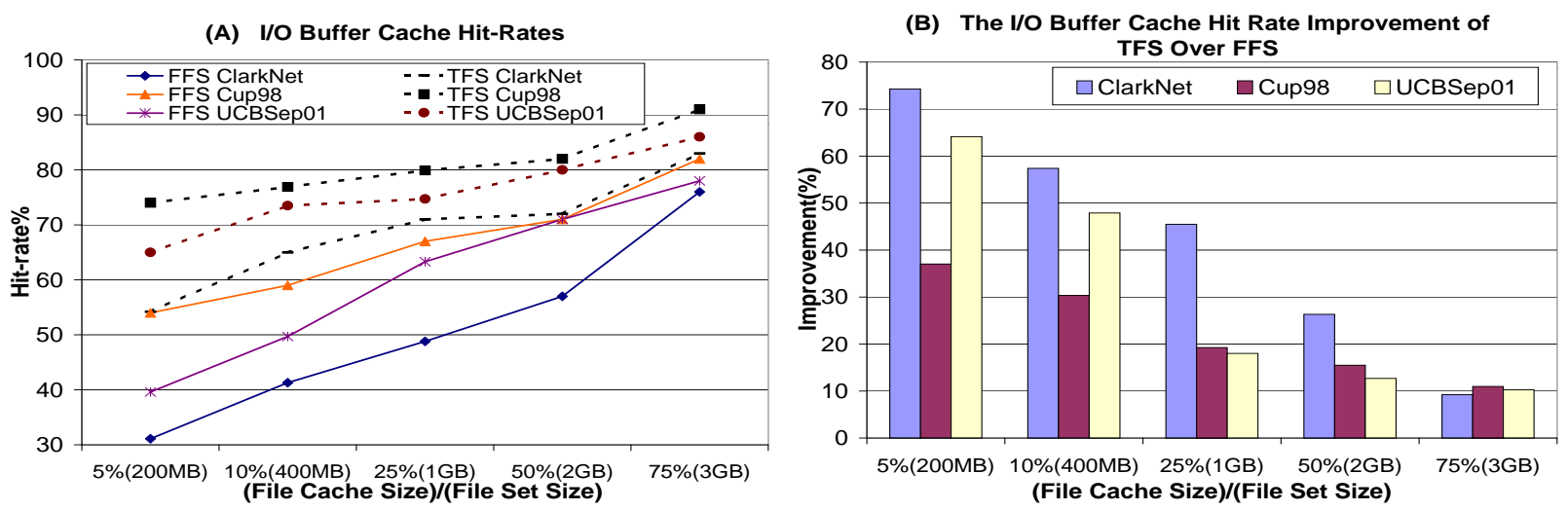

Figure 6: Buffer cache hit rates between TFS and FFS

lation confirms that TFS can drastically improve large Web server performance. By running one large real-world trace and two pseudo traces derived from real-world traces, we found that, TFS improves the system throughput by $20 \%$ to $160 \%$ and reduces the disk I/Os of FFS -async by 18 $77 \%$ when the buffer cache size is varied from $200 \mathrm{MB}$ to 3 GB. For future work, we are trying to improve the localitygrouping algorithms in order to further improve the cache hit rates. We will also explore the possibilities of applying TFS to other Web-related platforms such as mail servers.

\section{References}

[1] K. Kant and Y. Won., 'Performance impact of uncached fi le accesses in specweb99," in Proceedings of 2nd IEEE Workshop on workload characterization, (Austin TX), Oct 1999.

[2] P. Barford and M. Crovella, "A performance evaluation of hyper text transfer protocols," in Proceedings of the ACM SIGMETRICS International Conference on Measurement and Modeling of Computing Systems (SIGMETRICS99), vol. 27,1 of SIGMETRICS Performance Evaluation Review, (New York), pp. 188-197, ACM Press, May 1-4 1999.
[3] O. A. Adebowale, 'Impact of document caching on web server workloads," tech. rep., University of Saskatchewan, 1997. citeseer.nj.nec.com/408844.html.

[4] P. Barford, A.Bestavros, A.Bradley, and M.E.Crovella, 'Changes in web client access patterns: characteristics and caching implications, world wide web." Special issue on Characterization and Performance Evaluation, 1999.

[5] M. F. Arlitt and C. L. Williamson, "Internet Web servers: workload characterization and performance implications," IEEE/ACM Transactions on Networking, vol. 5, no. 5, pp. 631-645, 1997.

[6] M. A. Tai, 'Workload characterization of the 1998 world cup web site,” Tech. Rep. HPL-1999-35R1, HP, 1999.

[7] M. Rosenblum and J. K. Ousterhout, "The design and implementation of a log-structured fi le system," ACM Transactions on Computer Systems, vol. 10, pp. 26 - 52, Feb. 1992.

[8] Y. Hu and Q. Yang, 'DCD—disk caching disk: A new approach for boosting I/O performance," in Proceedings of the 23rd International Symposium on Computer Architecture (ISCA'96), (Philadelphia, Pennsylvania), pp. 169-178, May 1996. 
[9] G. R. Ganger, M. K. McKusick, C. A. N. Soules, and Y. N. Patt, 'Soft updates: a solution to the metadata update problem in file systems," ACM Transactions on Computer Systems, vol. 18, pp. 127-153, May 2000.

[10] C. R.Lumb, J. Schindler, G. R. Ganger, and D. F. Nagle, "Towards higher disk head utilization: Extracting free bandwidth from busy disk drives," in Proceedings of the 2000 Conference on Operating System Design and Implementation (OSDI), (San Diego), Oct. 2000.

[11] D. Roselli, J. R. Lorch, and T. E. Anderson, "A comparison of fi le system workloads," in Proceedings of the 2000 USENIX Conference, June 2000.

[12] P. Cao and S. Irani, "Cost-aware WWW proxy caching algorithms," in Proceedings of the USENIX Symposium on Internet Technologies and Systems (ITS-97), (Berkeley), pp. 193206, USENIX Association, Dec. 8-11 1997.

[13] M. Crovella and A. Bestavros, 'Self-similarity in WorldWide Web traffi c evidence and possible causes," in Proceedings of the SIGMETRICS '96 conference, May 1996. http://cswww.bu.edu/faculty/best/res/papers/sigmetrics96.ps.

[14] J. Ousterhout and F. Douglis, 'Beating the I/O bottleneck: A case for log-structured fi le systems," tech. rep., Computer Science Division, Electrical Engineering and Computer Sciences, University of California at Berkeley, Oct. 1988.

[15] G. R. Ganger, B. L. Worthington, and Y. N. Patt, The DiskSim Simulation Environment Version 2.0 Reference Мапиа. CMU/University of Michigan, December 1999.

[16] Lawrence Berkeley National Laboratory, "The internet traffi c archive," Apr. 2000. http://ita.ee.lbl.gov/index.html.

[17] The Internet Engineering Task Force, "The HTTP 1.1 Protocol-Draft," 1997. http://www.ietf.org.

[18] J. Wang, R. Min, Y. Zhu, and Y. Hu, 'UCFS - a user-space, high performance, customized fi le system for web proxy servers," IEEE transactions on computers, vol. 51, pp. 10561073, September 2002.

[19] E. Shriver, E. Gabber, L. Huang, and C. A. Stein, 'Storage management for web proxies," in Proceedings of the 2001 USENIX Annual Technical Conference (USENIX-01), (Berkeley, CA), pp. 203-216, The USENIX Association, June 25302001.

[20] C. Maltzahn, K. J. Richardson, and D. Grunwald, 'Reducing the disk $\mathrm{I} / \mathrm{O}$ of web proxy server caches," in Proceedings of the 1999 USENIX Annual Technical Conference (USENIX99), (Berkeley, CA), pp. 225-238, USENIX Association, June 6-11 1999.

[21] A. Iyengar, S. Jin, and J. Challenger, 'Effi cient algorithms for persistent storage allocation," in Proceedings of the 18th of IEEE Symposium on Mass Storage Systems, (San Diego, California), April 2001.

[22] M. Kaashoek, D. Engler, G. Ganger, and D. Wallach, "Server operating systems," in 1996 SIGOPS European Workshop., (Connemara,Ireland), 1996.

[23] A. S. Tanenbaum, R. van Renesse, H. van Staveren, G. J. Sharp, S. J. Mullender, J. Jansen, and G. van Rossum, 'Experiences with the Amoeba distributed operating system," Communications of the ACM, vol. 33, no. 12, pp. 46-63, 1990.
[24] M. K. McKusick, M. J. Karels, and K. Bostic, "A pageable memory based fi lesystem," in Proceedings of the Usenix Summer 1990 Technical Conference, (Berkeley, CA, USA), pp. 137-144, Usenix Association, June 1990.

[25] P. Snyder, “tmpfs: A virtual memory fi le system," in Proceedings of the Autumn 1990 European UNIX Users' Group Conference, pp. 241-248, Oct. 1990. 